\title{
Improved Accuracy of Heteronuclear Transverse Relaxation Time Measurements in Macromolecules. Elimination of Antiphase Contributions*
}

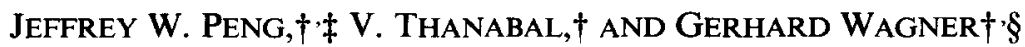 \\ †Department of Biological Chemistry and Molecular Pharmacology, Harvard Medical School, \\ 240 Longwood Avenue, Boston, Massachusetts 02115; and $\ddagger$ Biophysics Research Division, \\ University of Michigan, 2200 Bonisteel Boulevard, Ann Arbor, Michigan 48109
}

Received June 6, 1991

Heteronuclear $T_{2}$ relaxation measurements $\left({ }^{13} \mathrm{C},{ }^{15} \mathrm{~N}\right)$ play an integral role in the characterization of internal motions in proteins by NMR (1-3). Recently there has been some controversy concerning the correct method of measuring ${ }^{15} \mathrm{~N}$ or ${ }^{13} \mathrm{C} T_{2}$ values because different experiments produced different (mainly too short) $T_{2}$ values $(1-3)$. We propose and show experimental evidence that this is due to an oscillation between in-phase and antiphase coherence of the heteronucleus if chemical-shift refocusing and concomitant proton decoupling are achieved via a single (Carr-Purcell) or a train of (Meiboom-Gill) X-nucleus $180^{\circ}$ pulses. This leads to a mixture of contributions from in-phase and antiphase relaxation, and the latter causes apparently faster $T_{2}$ relaxation. We propose to eliminate the antiphase contributions from the $T_{2}$ measurements by applying a spin lock on the heteronucleus. The result is a significant improvement in the accuracy of heteronuclear $T_{2}$ measurements.

A basic 2D pulse sequence for measuring heteronuclear $T_{2}$ values in a $2 \mathrm{D}$ NMR experiment ( 1 ) is shown in Fig. 1a. We are mainly concerned with ${ }^{15} \mathrm{~N}$ relaxation and denote the heteronucleus as $\mathrm{N}$ and follow the product-operator formalism (4). In the pulse sequence of Fig. 1a, in-phase transverse coherence, $\mathrm{N}_{x}$, relaxes during a refocusing delay $\tau$, primarily due to dipole-dipole interactions between the $\mathrm{N}$ spins and their directly bonded protons (I spins). ${ }^{15} \mathrm{~N}$ relaxation studies on the proteinase inhibitor eglin $\mathrm{c}$ reveal that the use of different refocusing pulse schemes (Fig. la: $i$, ii, iii) yields different transverse relaxation times (5). Specifically, the use of a single $180^{\circ}$ pulse produces, in small proteins, relaxation times about $50 \%$ shorter than those obtained using a $3 \mathrm{kHz}$ spin lock. Carr-Purcell-Meiboom-Gill (CPMG) $(6,7)$ relaxation times yield intermediate values, and the apparent $T_{2}$ values increase as the spacing between the $180^{\circ}$ pulses is narrowed. However, with our spectrometer, we never came closer than to about $75 \%$ of the spin-lock values, being limited by a hard-pulse duty cycle of $2 \%$. For all residues in the protein, the maximum transverse relaxation time is

* The main ideas of this manuscript were presented at the Keystone Symposium "Frontiers of NMR Spectroscopy in Molecular Biology," April 8-13, 1991.

$\S$ To whom correspondence should be addressed. 
a.)

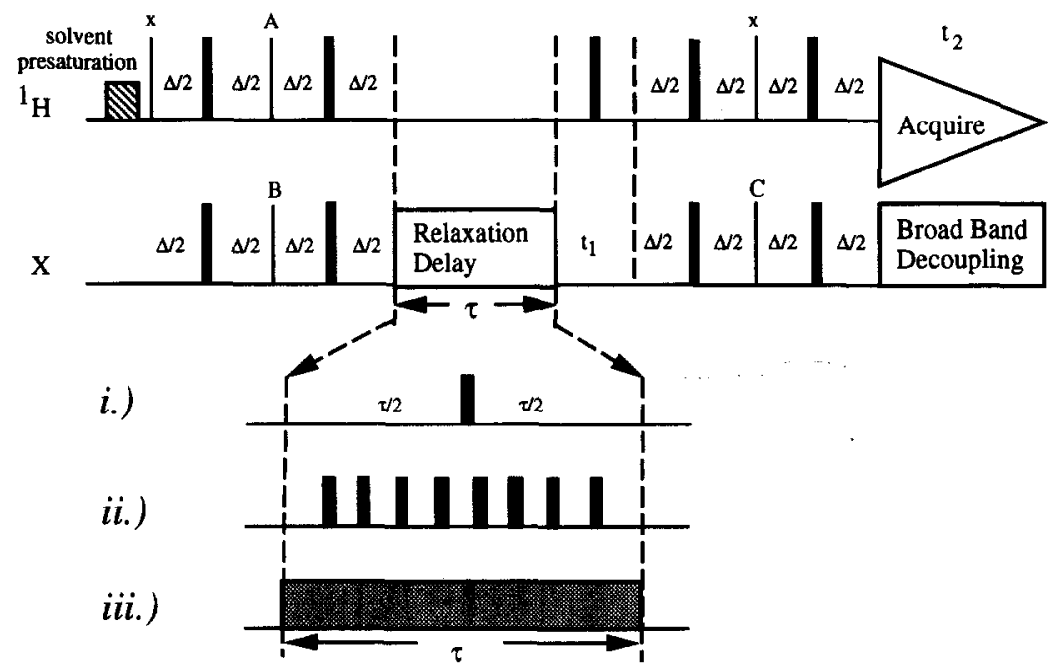

b.)

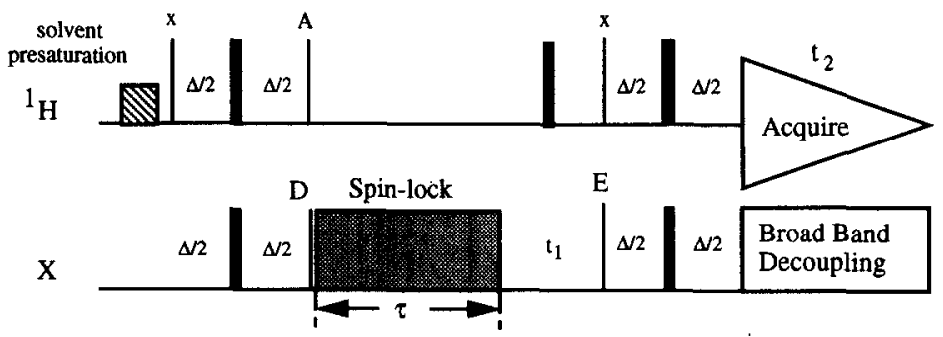

Fig. 1. (a) Two-dimensional pulse sequence for measuring heteronuclear $T_{2}$ values. The results are ${ }^{1} \mathrm{HX}$ correlation spectra with cross-peak intensities determined by the extent of transverse relaxation during the period $\tau ; 90^{\circ}$ and $180^{\circ}$ pulses are indicated by thin and thick vertical bars, respectively. The delays $\Delta / 2$ are set to $1 / 4 J_{\mathrm{XH}}$. Pulse schemes for refocusing transverse $\mathrm{X}$-nucleus magnetization during the relaxation delay include (i) a single $180^{\circ}$ refocusing pulse, (ii) a CPMG pulse train, and (iii) a weak on-resonance spin lock. CPMG trains consisted of $180^{\circ}$ pulses of $100 \mu$ s separated by delays. The spin lock consisted of contiguous $180^{\circ}$ pulses of $174 \mu \mathrm{s}$ without separating delays. Phase cycling is as follows: (A) $4(+y) 4(-y)$, (B) $+x$, $-x,(\mathrm{C}) 2(+x) 2(-x)+$ TPPI. The receiver phases are $(-x, x, x,-x, x, x, x, x)$. (b) Two-dimensional pulse sequence for measuring spin-locked antiphase relaxation. The spin lock is of the same type as that in a. Additional phase cycling includes (D) $+y,-y$, (E) $2(+y) 2(-y)+$ TPPI. Spectra were recorded on both Bruker AMX500 and GE Omega 500 spectrometers.

observed using the spin lock. Therefore, it is unlikely that chemical-exchange phenomena as proposed previously (2) are responsible for the short $T_{2}$ values in the single $180^{\circ}$ pulse scheme, since exchange processes are expected to be localized in certain regions of the protein structure. Note that the spin-lock relaxation times are rigorously heteronuclear $T_{1 \rho}$ values. The utility of $T_{1 \rho}$ as another means of measuring $T_{2}$ in liquids was first described by Redfield $(8)$ and later by Freeman and Hill $(9)$. For resonances at the spin-lock frequency, $T_{1 \rho}$ is equal to $T_{2}$, provided the protein behaves approximately as a rigid, spherically symmetric tumbler with a rotational correlation time, $\tau_{\mathrm{c}}$, much less than reciprocal of the spin-lock field strength. Thus, for spin-lock 
strengths in the kilohertz range, we demand $\tau_{\mathrm{c}} \ll 10 \mu \mathrm{s} / \mathrm{rad}$; this is satisfied by proteins that have $\tau_{\mathrm{c}}$ in the nanosecond regime $(2,3,5)$. More detailed aspects of $T_{\mathrm{l} \rho}$ are given elsewhere (5). For resonances away from the spin-lock carrier frequency, the ratio of $T_{1 \rho}$ to $T_{2}$ is approximately proportional to $1 / \sin ^{2} \beta$, where $\beta$ is the tip angle of the effective field in the rotating frame. For example, $a^{15} \mathrm{~N}$ sweep width of $\pm 700 \mathrm{~Hz}(1.4$ $\mathrm{kHz}$ ) has tip angles of $77^{\circ}$ at the edges of the spectrum using a $3 \mathrm{kHz}$ spin lock. $T_{1_{\rho}}$ is then expected to be about $5 \%$ greater than $T_{2}$. The typical precision of the $T_{2}$ measurements is such that these overestimations are not deleterious to subsequent motional interpretation of the data. In our studies at $11.7 \mathrm{~T}$ all of the amide ${ }^{15} \mathrm{~N}$ signals were contained within a sweep width of $1200 \mathrm{~Hz}$. Thus, the spin lock can be reasonably approximated as on-resonance for all of these cross peaks. For much wider heteronuclear spectral widths, severe off-resonance effects can be eliminated simply by varying the spin-lock carrier position to complete the coverage of the desired spectral range.

We propose that the evolution of in-phase $\mathrm{N}_{x}$ coherence into antiphase $2 \mathrm{I}_{z} \mathrm{~N}_{y}$ coherence is the primary cause for the shorter $T_{2}$ values in the single $180^{\circ}$ pulse experiment. Although $\mathrm{N}_{x}$ is refocused at the end of the $(\tau / 2)-180^{\circ}-(\tau / 2)$ period, the net coherence oscillates between $\mathrm{N}_{x}$ and antiphase $2 \mathrm{I}_{z} \mathrm{~N}_{y}$ coherence during either half of the $\tau$ period. Accordingly, the relaxation times of both in-phase and antiphase coherence must be considered. The relaxation time of antiphase coherence, $T_{2 z}$, is significantly shorter than $T_{2}$ for proteins, due to ${ }^{1} \mathrm{H}-{ }^{1} \mathrm{H}$ dipolar interactions experienced by the protons directly bonded to the $\mathrm{X}$ nuclei. Since the apparent transverse relaxation time reflects a mixture of both $T_{2}$ and $T_{2 z}$, an erroneously short " $T_{2}$ " will be recorded.

The faster relaxation of antiphase coherence as compared to in-phase coherence has been established in the literature $(10-12)$. For example, Vold and Vold $(10)$ discuss the effects of random fields at the I spin upon the relaxation rates of transverse $\mathrm{N}$-spin coherences. For our case, these random fields are due to ${ }^{1} \mathrm{H}-{ }^{1} \mathrm{H}$ dipolar interactions which induce transitions in the I spins. We briefly highlight the causes for the faster relaxation here. If we first consider an isolated IN two-spin system, then antiphase (i.e., $2 \mathrm{I}_{z} \mathrm{~N}_{y}, 2 \mathrm{I}_{z} \mathrm{~N}_{x}$ ) coherence decays as (13-15)

$$
\frac{d\left\langle 2 I_{z} N_{(y, x)}\right\rangle}{d t}=-\frac{1}{T_{2 z(\text { isol })}}\left\langle 2 I_{z} N_{(y, x)}\right\rangle,
$$

where

$$
\frac{1}{T_{2 z(\text { isol })}}=\frac{\left(h \gamma_{\mathrm{I}} \gamma_{\mathrm{N}}\right)^{2}}{8 r_{\mathrm{IN}}^{6}}\left\{4 J(0)+J\left(\omega_{\mathrm{I}}-\omega_{\mathrm{N}}\right)+3 J\left(\omega_{\mathrm{N}}\right)+6 J\left(\omega_{\mathrm{I}}+\omega_{\mathrm{N}}\right)\right\} .
$$

This can be compared to familiar expression for transverse relaxation rate of in-phase coherence

$$
\frac{1}{T_{2}}=\frac{\left(\hbar \gamma_{\mathrm{I}} \gamma_{\mathrm{N}}\right)^{2}}{8 r_{\mathrm{IN}}^{6}}\left\{4 J(0)+J\left(\omega_{\mathrm{I}}-\omega_{\mathrm{N}}\right)+3 J\left(\omega_{\mathrm{N}}\right)+6 J\left(\omega_{\mathrm{I}}\right)+6 J\left(\omega_{\mathrm{I}}+\omega_{\mathrm{N}}\right)\right\} .
$$

The spectral density function, $J(\omega)$, becomes a Lorentzian distribution,

$$
J(\omega)=\frac{2}{5} \frac{\tau_{\mathrm{c}}}{1+\left(\omega \tau_{\mathrm{c}}\right)^{2}}
$$


if we model the protein as an isotropically tumbling rigid body with a rotational correlation time, $\tau_{\mathrm{c}}$. Note from Eq. [2] that $1 / T_{2 z \text { (isol) }}$ is identical to $1 / T_{2}$ except that it lacks a $6 J\left(\omega_{1}\right)$ term. Therefore, if one considers only dipole-dipole interactions between the $\mathrm{I}$ and $\mathrm{N}$ spins, the antiphase relaxation times would actually be longer than $T_{2}$. However, in proteins $J\left(\omega_{\mathrm{I}}\right)$ may be very small. More importantly, the proton directly bonded to the $\mathrm{X}$ nucleus ( $\mathrm{I}_{i}$ spins $)$ can enjoy significant spin-lattice relaxation due to other nonbonded protons ( $\mathrm{I}_{j}$ spins) within about $5 \AA$ distance. When we include the effects of $\mathrm{I}_{i}-\mathrm{I}_{j}$ dipole-dipole interactions, the antiphase coherence for the $i$ th IN bond decays as

$$
\frac{d\left\langle 2 I_{i z} N_{(y, x)}\right\rangle}{d t}=-\frac{1}{T_{2 z}}\left\langle 2 I_{i z} N_{(y, x)}\right\rangle-\sum_{j \neq i} \sigma_{i j}\left\langle 2 I_{j z} N_{(y, x)}\right\rangle,
$$

where

$$
\frac{1}{T_{2 z}}=\frac{1}{T_{2 z(\text { isol })}}+\sum_{j \neq i} \rho_{i j}
$$

and

$$
\begin{aligned}
& \rho_{i j}=\frac{\hbar^{2} \gamma_{\mathrm{I}}^{4}}{4 r_{i j}^{6}}\left\{J\left(\omega_{\mathrm{I} i}-\omega_{\mathrm{I} j}\right)+3 J\left(\omega_{\mathrm{I} i}\right)+6 J\left(\omega_{\mathrm{I} i}+\omega_{\mathrm{I} j}\right)\right\} \\
& \sigma_{i j}=\frac{\hbar^{2} \gamma_{\mathrm{I}}^{4}}{4 r_{i j}^{6}}\left\{6 J\left(\omega_{\mathrm{I} i}-\omega_{\mathrm{Ij}}\right)-J\left(\omega_{\mathrm{I} i}-\omega_{\mathrm{I} j}\right)\right\} .
\end{aligned}
$$

The quantity $1 / T_{2 z \text { (isol) }}$ is the antiphase relaxation time for an isolated IN spin pair given in Eq. [2]. The expression given in Eq. [5] shows that the direct relaxation rate is increased from $1 / T_{2 z \text { (isol) }}$ in the simpler IN case to $1 / T_{2 z}^{\prime}=1 / T_{2 z \text { (isol) }}+\sum_{j \neq i} \rho_{i j}$. The $\rho_{i j}$ terms are the constituent spin-lattice relaxation rates of the directly bonded proton $\mathrm{I}_{i}$, due to dipole-dipole interactions with nonbonded protons $\mathrm{I}_{j}(15,16)$. The $\sigma_{i j}$ terms indicate cross-relaxation pathways $(15,16)$ to antiphase coherences involving spins that do not experience the one-bond heteronuclear coupling, $J_{\mathrm{IN}}$. As such, these coherences cannot refocus and are not detected in the 2D experiments described here (Fig. 1). In-phase $\mathrm{N}_{x}$ coherence is not relaxed by the ${ }^{1} \mathrm{H}-{ }^{1} \mathrm{H}$ dipolar interactions and therefore $T_{2 z}$ is significantly shorter than $T_{2}$. This fact can be understood by recalling that the $\mathrm{N}$-spin transverse coherence can be resolved into two component coherences corresponding to the $\mathrm{I}_{i}$ spin up and spin down, respectively. These components have the same phase in the transverse plane in the case of $\mathrm{N}_{x}$ and are $180^{\circ}$ opposed in the case of $2 \mathrm{I}_{i z} \mathrm{~N}_{(y, x)}$. However, the $\mathrm{I}_{i}$ spin flips caused by $\mathrm{I}_{i}-\mathrm{I}_{j}$ dipolar interactions connect only $\mathrm{N}$-spin coherences with the same phase. Thus, the net amount of in-phase $\mathrm{N}_{x}$ coherence is unaffectcd, while the net amount of antiphase coherence is decreased. In contrast, $\mathrm{I}_{i}$ spin flips caused by $\mathrm{I}_{i}-\mathrm{N}$ dipolar interactions invert the phase of the $\mathrm{N}$ spin coherences as a result of the spin operators $I_{i} \pm N_{z}$ in the dipolar Hamiltonian; thus $1 / T_{2 z \text { (isol) }}$ is independent of the $I_{i}$ transition frequency, $\omega_{1}$.

Note that the present explanation for the artificially short $T_{2}$ values emphasizes two steps: coherent antiphase evolution followed by antiphase relaxation. This contrasts to alternative explanations which blame the short $T_{2}$ values on scalar relaxation of 
the second kind (2). Scalar relaxation of the second kind, as described by Abragam (13), results from the random modulation of the IN scalar interaction, due to rapid relaxation of the I spin. The rapid relaxation forces the collapse of the $\mathrm{N}$-spin doublet. Thus, in order for scalar relaxation of the second kind to be applicable, we require the $\mathrm{I}_{i}$-spin (proton) $T_{1}$ values to satisfy $T_{1} \ll 1 / 2 \pi J_{\mathrm{IN}}$ (about $1.77 \mathrm{~ms} / \mathrm{rad}$ for ${ }^{15} \mathrm{~N}-$ ${ }^{1} \mathrm{H}$ bonds). Since the proton $T_{1}$ values are at least on the $100 \mathrm{~ms}$ time scale, an explanation of the apparently short $T_{2}$ values cannot be given in terms of scalar relaxation of the second kind.

Clearly, to obtain correct $T_{2}$ measurements, it is necessary to suppress the antiphase evolution during the transverse relaxation delay. This is precisely the effect of the lowpower spin lock shown in the pulse sequence of Fig. 1a(iii). The CPMG method also suppresses antiphase evolution, although the extent of suppression depends on the spacing between the $180^{\circ}$ pulses. Specifically, the $\tau$ periods between $180^{\circ}$ refocusing pulses must satisfy $\tau \ll 1 / 2 J_{\mathrm{IN}}$. For ${ }^{15} \mathrm{~N}-{ }^{1} \mathrm{H}$ spin systems, $J_{\mathrm{IN}}$ is about $90 \mathrm{~Hz}$; thus, $\tau$ should be well below $5.5 \mathrm{~ms}$. However, such short $\tau$ periods demand significant duty cycles at high power on the X-nucleus channel and may be unfeasible for some spectrometers. If sufficiently short $\tau$ values cannot be implemented, then artificially short $T_{2}$ values will be recorded. Moreover, the recorded values will depend on the choice of pulse spacing $\tau$. In our studies, we were limited to $180^{\circ}{ }^{15} \mathrm{~N}$ pulses of $100 \mu \mathrm{s}$ and $\tau=5 \mathrm{~ms}$ ( $2 \%$ duty cycle), resulting in the uniformly shorter $T_{2}$ values reported above. Thus, in these situations, the use of a low-power spin lock is a superior alternative to the CPMG method for obtaining accurate $T_{2}$ measurements.

To confirm that antiphase evolution and relaxation are responsible for the short $T_{2}$ measurements, we have performed both spin-locked in-phase and spin-locked antiphase ${ }^{15} \mathrm{~N}$-relaxation experiments on the protein eglin $\mathrm{c}$. The antiphase experiment shown in Fig. Ib simply applies the spin lock after the initial creation of antiphase $2 \mathrm{I}_{i z} \mathrm{~N}_{x}$ coherence. Under the spin-lock and resonance-offset provisions given above, these experiments provide $T_{2}$ and $T_{2 z}$ values, respectively. Note that the spin locking prevents the mixing of in-phase and antiphase magnetizations in both sequences; thus, $T_{2}$ and $T_{2<}$ are measured separately. The resulting relaxation times clearly show that $T_{2}$ is significantly longer than $T_{2 z}$ for all residues in eglin c. Examples of the comparative $T_{2}$ and $T_{2 z}$ fits are shown as upper and lower curves, respectively, in Fig. 2 for the amide ${ }^{15} \mathrm{~N}$ nuclei of Val 14 and Arg 53.

In conclusion, the correct measurement of heteronuclear $T_{2}$ values demands that the in-phase transverse coherence be prevented from evolving into antiphase coherence. Failure to do this will produce erroneously short transverse relaxation times, since antiphase magnetization experiences significant proton-proton relaxation. If spectral density $J(\omega)$ is Lorentzian and the spin lock is sufficiently on-resonance, then $T_{1 \rho}$ $=T_{2}$ and a spin-lock series can be used to measure the heteronuclear $T_{2}$ values. Alternatively, the CPMG method can be used if the pulse spacing is sufficiently narrow. However, if the latter method is used, additional experiments should be run to ensure that the observed $T_{2}$ values do not increase significantly as the pulse spacing is narrowed. The advantage of the spin-lock experiment shown in Fig. 1a(iii) is that the relaxation times obtained do not suffer from the ambiguity associated with this spacing between refocusing pulses. 
a.) Val 14

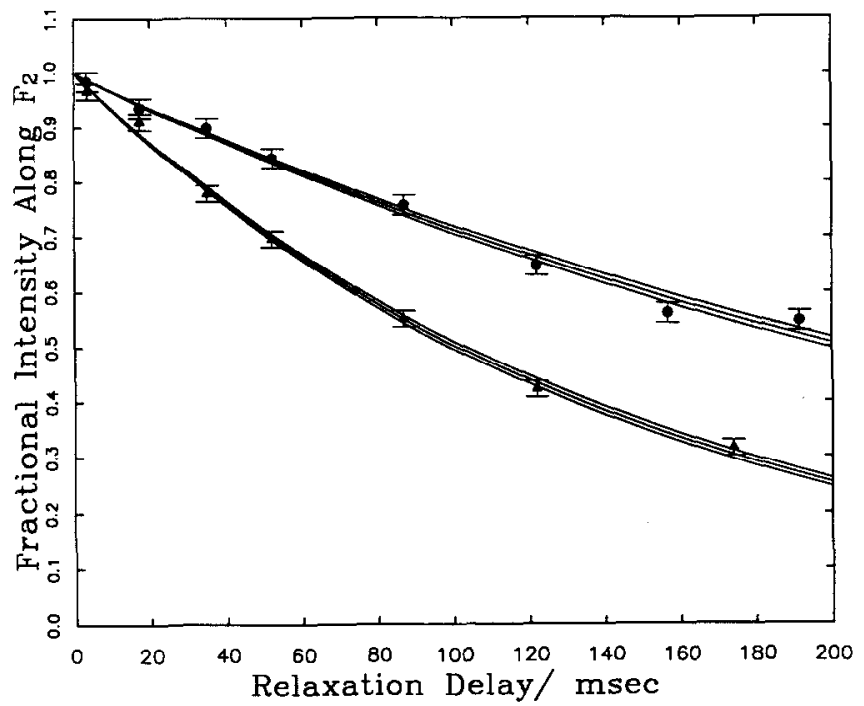

b.) Arg 53

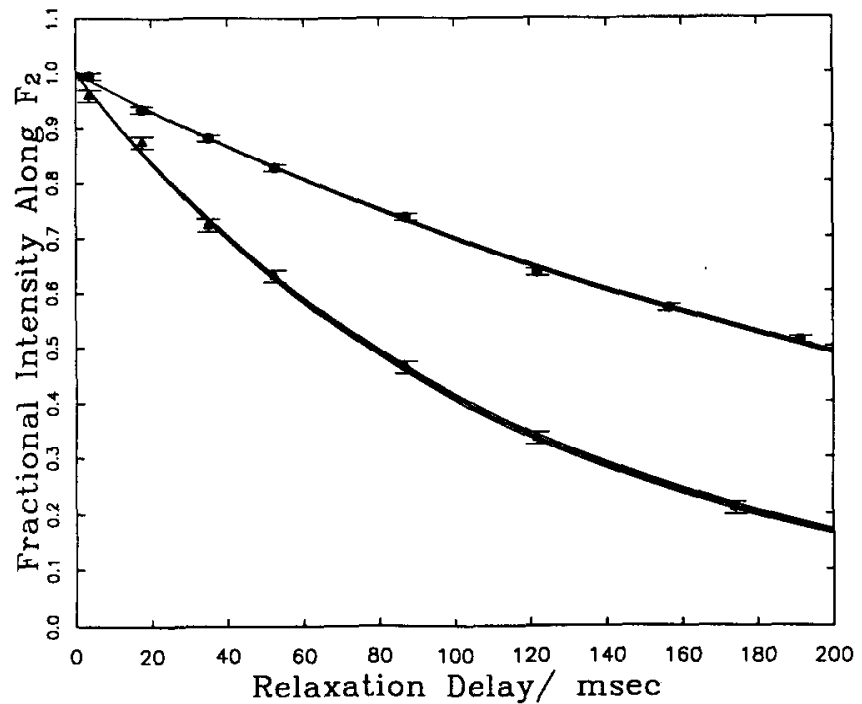

FIG. 2. Examples of comparative $T_{2}$ and $T_{2 z}$ fits for residues Val 14 and Arg 53 of the proteinase inhibitor eglin c. $T_{2}$ and $T_{2 z}$ values were measured using the spin-lock pulse sequences in Figs. la and $\mathrm{lb}$. Relaxation times are extracted by fitting integrals of cross peaks along the $F_{2}\left({ }^{1} \mathrm{H}\right)$ dimension to an exponential decay using a nonlinear least-squares fitting procedure from the software package PLOT (New Unit Inc., Ithaca, New York). The $T_{2}$ fits are always the upper set of curves. (a) Val 14: $T_{2}=294 \pm 5 \mathrm{~ms}, T_{2 z}=146 \pm 3$ ms. (b) Arg 53: $T_{2}=278 \pm 3 \mathrm{~ms}, T_{22}=112 \pm 3 \mathrm{~ms}$. For each fit, three curves are plotted. The central curve corresponds to the fitted relaxation time while the bounding curves correspond to the quoted uncertainties. Error bars indicate the root-mean-square deviation from the fitted function to the peak intensities. 


\section{ACKNOWLEDGMENTS}

We thank Dr. Dirk Heinz and Dr. Marcus Grütter, Ciba-Geigy, Basel, Switzerland, for the gift of $\left[{ }^{15} \mathrm{~N}\right]$ eglin c. This work was supported by NSF Grant DMB-9007878 and NIH Grant T32-GM08270-03 to J.P.

\section{REFERENCES}

1. N. R. Nirmala AND G. WaGNer, J. Magn. Reson. 82, 659 (1989).

2. L. E. KAY, D. A. TORCHIA, AND A. BAX, Biochemistry 28, 8972 (1989).

3. G. M. Clore, P. C. Driscoll, P. T. Winghield, and A. M. Gronenborn, Biochemistry 29, 7387 (1990).

4. O. W. Sørensen, G. W. Eich, M. H. Levitt, G. Bodenhausen, AND R. R. ERnst, Prog. NMR Spectrosc. 16, 163 (1983).

5. J. W. Peng, V. Thanabal, and G. Wagner, J. Magn. Reson., in press.

6. H. Y. CARr and E. M. Purcell, Phys. Rev. 94, 630 (1954).

7. S. MEIBOOM AND D. Gill, Rev. Sci. Instrum. 29, 688 (1958).

8. A. G. RedField, Phys. Rev. 98, 1787 (1955).

9. R. Freeman and H. D. W. Hill, J. Chem. Phys. 55, 1985 (1971).

10. R. R. Vold and R. L. Vold, J. Chem. Phys. 64, 320 (1976).

11. R. E. LoNDON, J. Magn. Reson. 86, 410 (1990).

12. A. Bax, M. Ikura, L. E. Kay, D. A. Torchia, And R. Tschudin, J. Magn. Reson. 86, 304 (1990).

13. A. Abragam, "The Principles of Nuclear Magnetism," Chap. 8, Clarendon Press, Oxford, 1961.

14. R. R. ERnst, G. Bodenhausen, And A. Wokaun, "Principles of Nuclear Magnetic Resonance in One and Two Dimensions," Chap. 2, Clarendon Press, Oxford, 1987.

15. I. Solomon, Phys. Rev. 99, 559 (1955).

16. S. Macura AND R. R. ERNST, Mol. Phys. 41, 95 (1980). 